\title{
Oxaliplatin/Irinotecan/Bevacizumab Followed by Docetaxel/ Bevacizumab in Inoperable Locally Advanced or Metastatic Gastric Cancer Patients - AGMT_GASTRIC-3
}

\author{
EWALD WÖLL ${ }^{1}$, JOSEF THALER ${ }^{2}$, FELIX KEIL ${ }^{3}$, BIRGIT GRUENBERGER ${ }^{4}$, \\ MICHAEL HEJNA ${ }^{5}$, WOLFGANG EISTERER ${ }^{6}$, MICHAEL A. FRIDRIK ${ }^{7}$, HANNO ULMER $^{8}$, \\ VERA TROMMET $^{2}$, FLORIAN HUEMER ${ }^{9}$, LUKAS WEISS $^{9}$ and RICHARD GREIL $^{9}$ \\ ${ }^{I}$ St. Vinzenz Krankenhaus Betriebs GmbH, Zams, Austria; \\ ${ }^{2}$ Department of Internal Medicine IV, Klinikum Wels-Grieskirchen GmbH, Wels, Austria; \\ ${ }^{3} 3$ rd Medical Department, Hanusch Hospital, Vienna, Austria; \\ ${ }^{4}$ St. John of God's Hospital, Vienna, Austria; \\ ${ }^{5}$ Medical University of Vienna, Vienna, Austria; \\ ${ }^{6}$ Medical University Hospital Innsbruck, Innsbruck, Austria; \\ ${ }^{7}$ Kepler Universitätsklinikum GmbH, Med Campus III, Klinik für Interne, Linz, Austria; \\ ${ }^{8}$ Department of Medical Statistics, Informatics and Health Economics, \\ Innsbruck Medical University, Innsbruck, Austria; \\ ${ }^{9}$ IIIrd Medical Department at the Paracelsus Medical University Salzburg, \\ Salzburg Cancer Research Institute (SCRI), Cancer Cluster Salzburg (CCS), Salzburg, Austria
}

\begin{abstract}
Background/Aim: Although high response rates using the doublet-chemotherapy of oxaliplatin and irinotecan as well as its combination with cetuximab in advanced gastric cancer were shown in previous trials, time to progression was short, suggesting acquired chemotherapy resistance. Patients and Methods: Sequential chemotherapy (oxaliplatin and irinotecan followed by docetaxel) combined with bevacizumab was investigated in the GASTRIC-3 trial. Patients achieving at least stable disease were continued on maintenance bevacizumab. Results: Objective response rate was available in 33 patients: Complete response (CR) $12.1 \%$, partial response (PR) 39.4\%, stable disease (SD) $27.3 \%$. Median time to progression was 7.0 months (95\%CI=5.0-11.0) and median overall survival was 11 months (95\%CI=9.0-15.0). Of note, two patients continue to
\end{abstract}

This article is freely accessible online.

This trial is registered with ClinicalTrials.gov ID NCT00952003 and Eudract No. 2008-006128-79.

Correspondence to: Prim. Univ.-Doz. Dr. Ewald Wöll, St. Vinzenz Krankenhaus Betriebs GmbH, Sanatoriumstr, 43, 6511 Zams, Austria. Tel: +43 54426007421, Fax: +43 54426007420, e-mail: e.woell@krankenhaus-zams.at

Key Words: Gastric cancer, bevacizumab, maintenance. receive bevacizumab maintenance therapy for more than 5 years with ongoing CR. Conclusion: Combining sequential chemotherapy with oxaliplatin/irinotecan and docetaxel with bevacizumab followed by bevacizumab maintenance is feasible and clinically active in advanced gastric cancer.

Gastric cancer is still one of the leading causes of death from intestinal neoplasias. Survival of patients with gastric cancer is poor with an overall 5-year survival rate of less than $20 \%$, and gastric cancer should be viewed as systemic disease even at early stages. Second-generation treatment regimens show a response rate between $21 \%$ and $27 \%$ with a median survival of 6 to 8 months (1). However, chemotherapy has shown to provide a significant benefit in the quantity and quality of life over best supportive care alone. Whilst no combination treatment regimen is recognized as standard for gastric cancer, continuous infusion of 5-Fluorouracil (5-FU) or oral 5-FU prodrugs combined with cisplatin or oxaliplatin is currently considered as reference treatment worldwide in HER-2 negative gastric cancer (2). Several agents have recently emerged as potential new options for advanced gastric cancer. The triple combination of 5-FU with cisplatin or oxaliplatin and docetaxel is one of the most effective treatments but shows considerable toxicity (3).

Two cytotoxic compounds with different mechanisms of action and lack of cross-resistance between them have been proven clinically active in the treatment of advanced 
colorectal cancer patients: irinotecan and oxaliplatin. The synergism between topoisomerase I inhibitor and platinum salts has been shown to be due to the stabilisation of DNA platinum adducts, when cells were exposed to the topoisomerase I inhibitor after the platinum compound. Furthermore, the clinical experience with both drugs as single agents and in combination has shown a nonoverlapping toxicity profile.

The combination of oxaliplatin $85 \mathrm{mg} / \mathrm{m}^{2} \mathrm{q} 2 \mathrm{w}$ with irinotecan $125 \mathrm{mg} / \mathrm{m}^{2} \mathrm{q} 2 \mathrm{w}$ was well tolerated and no doselimiting toxicity was reported from several phase II trials (46 ). This schedule was chosen for the present study. Analysis of 40 assessable patients in the phase II study (AGMT_GASTRIC-1) showed that this regimen was generally well tolerated and feasible in an outpatient setting. Frequently reported adverse events (more than 20\%) were grade 1 or 2 and included neutropenia, thrombocytopenia, anaemia, nausea, diarrhea (20\% grade $1,7 \%$ grade 2$)$, alopecia, polyneuropathy (7\% grade 2 , no grade 3 ) and handfoot syndrome. Only 2 out of 40 patients experienced grade 3 toxicity (anaemia and reversible renal failure). Thirty-six patients were assessable for response with a more than $50 \%$ tumor reduction in 6 of $13(46 \%)$ patients (4). In view of the favourable response rates and toxicity profile, we considered this regimen to be ideal for further assessment in combination with a molecular targeting agent.

Median time to progression in the GASTRIC-1 trial was 5.3 months. Resistance to irinotecan/oxaliplatin therefore must occur prior to this time point. In the presented GASTRIC-3 trial, chemotherapy is switched after three months to docetaxel, a very effective drug in gastric cancer (3) with a different mode of action compared to oxaliplatin and irinotecan. An upfront triple chemotherapy combination however would substantially increase toxicity (3), therefore, a sequential approach was chosen.

Bevacizumab is a monoclonal antibody directed against the vascular endothelial growth factor (VEGF), a major mediator of angiogenesis. In gastric cancer first results of feasibility and activity of bevacizumab in combination with irinotecan and cisplatin have been presented (7) but primary endpoints in phase III trials have not been reached (8). These data however have not been published at the time of initiation of the AGMT_GASTRIC-3 trial. Since chemotherapy alone shows only minor improvement in overall survival in this entity the combination of chemotherapy with biologicals is warranted. In our preceding GASTRIC-2 trial we investigated irinotecan/oxaliplatin in combination with cetuximab in 51 patients with inoperable gastric cancer. Efficacy however was not increased compared with the historic control group in unselected patients (5). The consequent development of AGMT GASTRIC trials enables historical comparisons and could therefore be important for hypothesis generation in upcoming randomised phase II/III trials.

\section{Patients and Methods}

This is a non-randomised, multicentre, open-label, single-arm phase II study in patients with histologically proven, inoperable, locally advanced or metastatic gastric cancer. Eligible patients had received no previous chemotherapy or immune therapy for inoperable disease but prior perioperative chemotherapy or adjuvant chemo/ radiotherapy were allowed. Patients received oxaliplatin $85 \mathrm{mg} / \mathrm{m}^{2}$, irinotecan $125 \mathrm{mg} / \mathrm{m}^{2}$ and bevacizumab $5 \mathrm{mg} / \mathrm{kg}$ for three cycles (one cycle comprises of two applications every other week) followed by docetaxel $50 \mathrm{mg} / \mathrm{m}^{2}$ and bevacizumab $5 \mathrm{mg} / \mathrm{kg}$ for a further three cycles (one cycle comprises of two applications every other week). Patients achieving at least stable disease were continued on maintenance bevacizumab until disease progression or unacceptable toxicity.

Assessment of response was performed according to the RECIST 2.0 criteria. Response was assessed every 12 weeks and at the end of study, always in comparison with screening status and last staging.

The statistical analysis was performed on the intention-to-treat population. Progression-free (PFS) and overall survival (OS) were analysed using the Kaplan-Meier method. Beside the estimated survival rates, median times were reported for both PFS and OS. Response rates and the proportion of patients with side effects were given as absolute and relative frequencies. No statistical testing was applied. All analyses were performed descriptively.

The study was approved by the ethics committee of the provincial government of Innsbruck on June 22, 2009 (protocol number: UN 3578), and by the respective ethics committee of each of the participating centers. All patients had given their written informed consent.

The trial was sponsored by Arbeitsgemeinschaft Medikamentöse Tumortherapie (AGMT). Roche and Sanofi-Aventis provided financial support, but had no input or influence on statistical analysis, data interpretation or writing of the manuscript. All authors took final responsibility for the interpretive analyses and agreed to submit the manuscript for publication.

\section{Results}

Between Sep 2009 and Mar 2012, 42 patients were screened for eligibility and 40 have been enrolled (Figure 1). Two patients did not meet inclusion criteria.

During induction treatment 14 patients discontinued prematurely for reasons other than progression (Figure 1). Two patients are still receiving bevacizumab maintenance therapy. Table I summarises the baseline characteristics of the trial cohort.

The median age of patients was 63 years. At the time of trial entry, $65 \%$ of patients were in a good overall health with an ECOG performance score of 0 .

At the time of data cut off (August 19, 2016) the median observation time was 11.5 months (IQR=6.25-19.0). Fourteen patients completed six cycles of induction. The median number of induction cycles per patient was 5.0 $(\mathrm{IQR}=2.5-6.0)$, the median number of maintenance cycles was $6.0(\mathrm{IQR}=3.0-12.5)$.

Evaluation of objective response rate was available in 33 of 40 patients, 7 patients discontinued before first response 


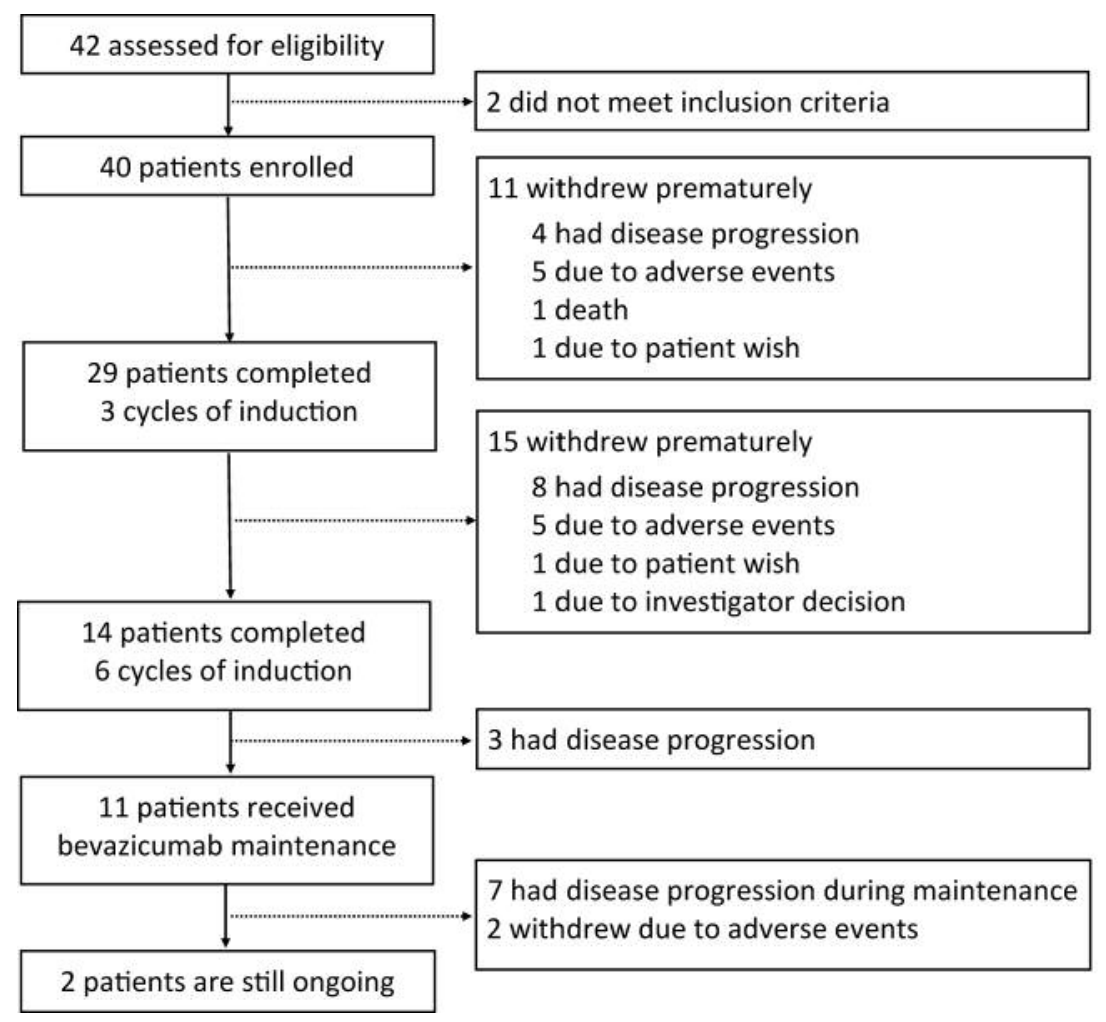

Figure 1. CONSORT flow diagram.

Table I. Patient demographics and baseline characteristics.

\begin{tabular}{lc}
\hline Baseline characteristics & \\
\hline Age (years, median, range) & $63(26-83)$ \\
Gender & \\
$\quad$ Male & $30 / 40(75.0 \%)$ \\
Female & $10 / 40(25.0 \%)$ \\
Body weight (kg, median, range) & $52(38-122)$ \\
ECOG PS & $26 / 40(65.0 \%)$ \\
0 & $12 / 40(30.0 \%)$ \\
1 & $2 / 40(5.0 \%)$ \\
Missing & $31(9-239)$ \\
Disease duration (days, median, IQR) & $12 / 40(30.0 \%)$ \\
Prior chemotherapy & \\
Metastatic disease & $26 / 40(65.0 \%)$ \\
Single* & $14 / 40(35.0 \%)$ \\
Multiple & \\
\hline
\end{tabular}

*Liver metastases in 11 patients, peritoneal metastases in 7 patients, lymph node metastases in 2 patients, lung metastases in 2 patients, ascites in 2 patients and other metastatic sites in 2 patients.

assessment for reasons other than progression. Four patients $(12.1 \%)$ showed a complete remission $(\mathrm{CR})$ and 13 patients $(39.4 \%)$ showed a partial remission (PR). Nine patients $(27.3 \%)$ had at least stable disease (SD) after three cycles.
Median time to progression was 7.0 months $(95 \% \mathrm{CI}=5.0$ $11.0)$ and median overall survival was 11 months (95\% CI=9.0-15.0) (Figure 2). Two patients are still receiving bevacizumab in continuing $\mathrm{CR}$ after more than 5 years of treatment.

Second-line treatment was applied in 23/34 patients (four patients already died during induction, Table II).

Overall, the combination of oxaliplatin/irinotecan and docetaxel with bevacizumab was well tolerated. Nevertheless, 12 patients discontinued due to drug-related toxicity. No treatment related deaths were reported.

Frequently reported adverse events (at least 20\% of patients) were gastrointestinal disorders, polyneuropathy, fatigue, neutropenia, hypokalaemia, hypertension and decreased appetite (Table III). Bevacizumab-associated sideeffects were rare: Grade 2 proteinuria occurred in three patients, arterial hypertension in 10 patients. One patient suffered from grade 3 gastric perforation and in three patients impaired wound healing was reported.

\section{Discussion}

First-line chemotherapy in inoperable or metastatic gastric cancer has shown a moderate, but significant improvement in overall survival and in some extent an improvement in 

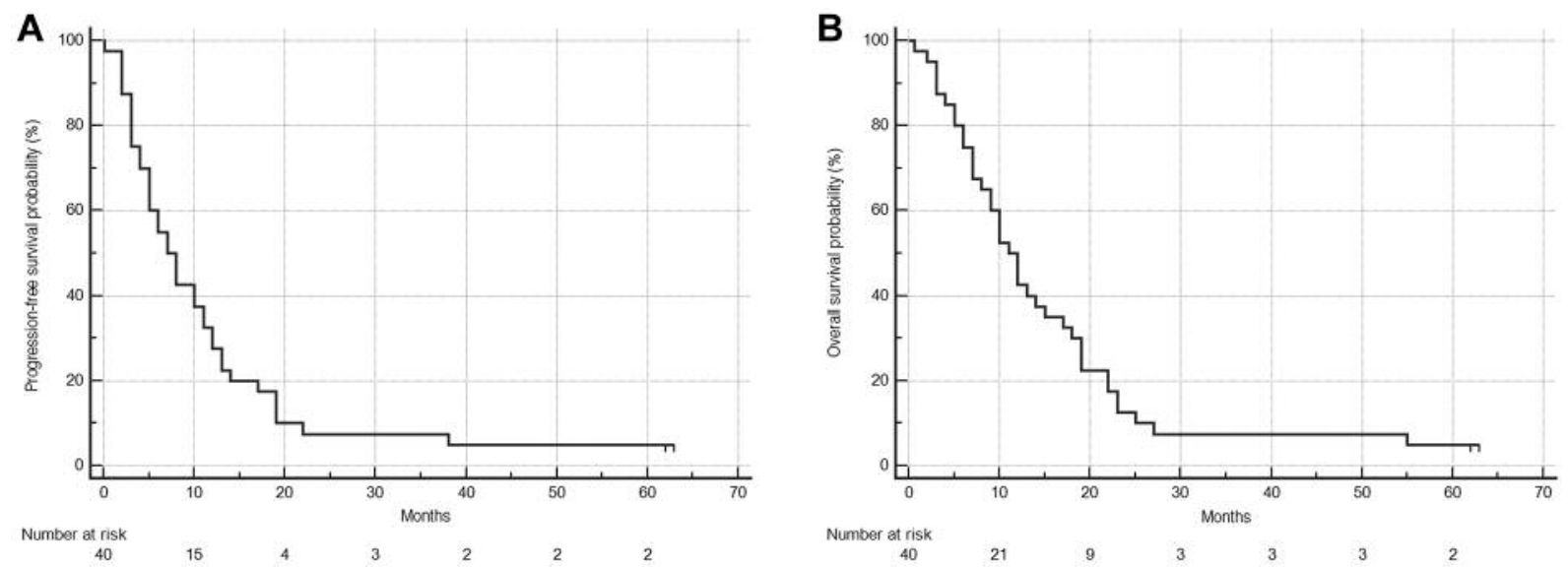

Figure 2. Kaplan-Meier estimates of progression-free survival (A), and overall survival (B).

Table II. Further treatment after progression.

\begin{tabular}{lc}
\hline Further treatment after progression & Patients* \\
\hline 5-FU/capecitabine based & 10 \\
Taxane based & 11 \\
Oxaliplatin/irinotecan reinduction & 9 \\
Other & 2 \\
\hline
\end{tabular}

*Eight patients had more than one treatment.

Table III. Incidence of adverse events, occurring in at least $20 \%$ of patients.

\begin{tabular}{lccc}
\hline Patients with adverse events & Grade 1-2 & Grade 3 & Grade 4 \\
\hline Diarrhea & $22(55.0)$ & $5(12.5)$ & 0 \\
Nausea & $22(55.0)$ & $1(2.5)$ & 0 \\
Abdominal pain/discomfort & $22(55.0)$ & $1(2.5)$ & 0 \\
Polyneuropathy & $20(50.0)$ & $1(2.5)$ & 0 \\
Fatigue & $17(42.5)$ & $1(2.5)$ & 0 \\
Vomiting & $16(40.0)$ & $1(2.5)$ & 0 \\
Neutropenia & $7(17.5)$ & $2(5.0)$ & $4(10.0)$ \\
Hypokalaemia & $7(17.5)$ & $2(5.0)$ & $1(2.5)$ \\
Hypertension & $10(25.0)$ & 0 & 0 \\
Decreased appetite & $8(20.0)$ & 0 & 0 \\
\hline
\end{tabular}

quality of life when compared to best supportive-care alone (2). A metaanalysis has confirmed this effect (9) and has shown that combined chemotherapy of at least two substances is superior to monochemotherapy $(\mathrm{HR}=0.83)$. Triple chemotherapy is even more effective but also more toxic. This treatment therefore is limited to fit patients and/or symptomatic patients in need of a rapid response $(2,10)$. Two randomised trials showed that oxaliplatin can substitute cisplatin without compromise in clinical efficacy and a different toxicity profile as well as some improvements in therapy application $(11,12)$. We, therefore, selected a sequential approach to reduce toxicity as compared to triple therapy. At time of initiation of the GASTRIC-3 trial, the data from the randomised phase III AVAGAST trial were not available (8) and addition of bevacizumab was highly promising in different tumor entities. In terms of the primary endpoint of overall survival, the results of the AVAGAST trial were not significant and the trial is therefore a formally negative trial. However, secondary endpoints (progressionfree survival (PFS) and response rates (RR)) were significant in favour of the bevacizumab-combination. The antiangiogenic approach furthermore prooved effective in two different phase III trials in second line treatment utilising ramucirumab $(13,14)$. Our data support further development of anti-angiogenic therapy since toxicity was tolerable and response rate as well as OS and PFS were promising given the fact that the patients treated showed unfavorable characteristics. All patients suffered from metastatic disease and almost one third of patients had received prior adjuvant or neoadjuvant chemotherapy. As compared with our historical control (4) the number of patients with CR, the overall response rate as well as PFS could be increased suggesting a higher efficacy of the approach investigated. A limitation of this trial is the fact that it remains unclear if the effect observed is achieved by the addition of bevacizumab or the switch in chemotherapy backbone.

Of special interest, however, is the fact that two patients still respond to treatment during bevacizumab maintenance therapy. Both patients showed a complete remission by radiographic assessment. One patient however still has viable 
tumor residues in subsequent gastroscopy biopsies underscoring the fact that bevacizumab monotherapy at least in this patient can control the disease. Arterial hypertension grade 2 observed during bevacizumab-treatment in this patient may be interpreted as a positive predictive factor for response to bevacizumab $(15,16)$.

A complete molecular workup of 295 chemotherapy naïve gastric adenocarcinomas has recently been published as part of the cancer genome atlas (17). By mutational analysis, analysis of methylation, amplification, mRNA, microRNA, and the proteome four different gastric cancer categories were defined. The Epstein-Barr virus associated subtype, the hypermutated subtype, the genomically stable subtype and a subtype with chromosomal instability. These subtypes might be important in the future to better predict not only prognosis of cancer patients but also response to certain treatments. Therefore, in-depth molecular workup was performed in the two long time responders analysing the coding sequence of 315 cancer-related genes and introns from 28 genes often rearranged or altered in solid tumors (FoundationOne ${ }^{\circledR}$, MA, USA). Unfortunately, no conformance could be elucidated. One patient showed a chromosomally instable subtype, the other patient was genomically stable.

In summary, more effort should be put into patient selection and identification of predictive markers. Unfortunately, our data could not elucidate a set of promising markers. The data, however, show that the sequential therapy of oxaliplatin, irinotecan in combination with bevacizumab followed by docetaxel in combination with bevacizumab followed by bevacizumab maintenance is feasible and active in patients suffering from metastatic gastric cancer. Two patients even showed long-term tumor remission for more than five years. Further evaluation of biomarkers is ongoing in order to eventually better characterise patients who are likely to respond to this therapeutic approach.

\section{Conflicts of Interest}

MF reports honoraria, consultant and advisory role and other support from Roche, RG reports grants and personal fees from Roche, LW reports travel support from Pfizer. WE, BG, FH, MH, FK, JT, VT, HU and EW declare no competing interests.

\section{References}

1 Howlader N NA, Krapcho M, Miller D, Bishop K, Altekruse SF, Kosary CL, Yu M, Ruhl J, Tatalovich Z, Mariotto A, Lewis DR, Chen HS, Feuer EJ, Cronin KA (eds). SEER Cancer Statistics Review, 1975-2013, National Cancer Institute. Bethesda, MD. 2016; http://seer.cancer.gov/csr/1975_2013/, based on November 2015 SEER data submission, posted to the SEER web site, June 2017.

2 Smyth EC, Verheij M, Allum W, Cunningham D, Cervantes A and Arnold D: Gastric cancer: ESMO Clinical Practice Guidelines for diagnosis, treatment and follow-up. Ann Oncol 27(suppl 5): v38-v49, 2016.
3 Van Cutsem E, Moiseyenko VM, Tjulandin S, Majlis A, Constenla M, Boni C, Rodrigues A, Fodor M, Chao Y, Voznyi E, Risse ML, Ajani JA and V325 Study Group: Phase III study of docetaxel and cisplatin plus fluorouracil compared with cisplatin and fluorouracil as first-line therapy for advanced gastric cancer: a report of the V325 Study Group. J Clin Oncol 24(31): 4991-4997, 2006.

4 Woll E, Kuhr T, Eisterer W, Gattringer K, Greil R, Zabernigg A, Hilbe W and Thaler J: Biweekly oxaliplatin and irinotecan chemotherapy in advanced gastric cancer. A first-line multicenter phase II trial of the Arbeitsgemeinschaft Medikamentose Tumortherapie (AGMT). Anticancer Res 28(5B): 2901-2905, 2008.

5 Woll E, Greil R, Eisterer W, Bechter O, Fridrik MA, Grunberger B, Zabernigg A, Mayrbäurl B, Russ G, Dlaska M, Obrist P and Thaler J: Oxaliplatin, Irinotecan and Cetuximab in Advanced Gastric Cancer. A Multicenter Phase II Trial (Gastric-2) of the Arbeitsgemeinschaft Medikamentose Tumortherapie (AGMT). Anticancer Res 31(12): 4439-4443, 2011.

6 Souglakos J, Syrigos K, Potamianou A, Polyzos A, Boukovinas I, Androulakis N, Kouroussis Ch, Vardakis N, Christophilakis Ch, Kotsakis A and Georgoulias V: Combination of irinotecan (CPT-11) plus oxaliplatin (L-OHP) as first-line treatment in locally advanced or metastatic gastric cancer: a multicentre phase II trial. Ann Oncol 15(8): 1204-1209, 2004.

7 Shah MA, Ramanathan RK, Ilson DH, Levnor A, D'Adamo D, O'Reilly E, Tse A, Trocola R, Schwartz L, Capanu M, Schwartz GK and Kelsen DP: Multicenter phase II study of irinotecan, cisplatin, and bevacizumab in patients with metastatic gastric or gastroesophageal junction adenocarcinoma. J Clin Oncol 24(33): 5201-5206, 2006.

8 Van Cutsem E, de Haas S, Kang YK, Ohtsu A, Tebbutt NC, Ming Xu J, Peng Yong W, Langer B, Delmar P, Scherer SJ and Shah MA: Bevacizumab in combination with chemotherapy as first-line therapy in advanced gastric cancer: a biomarker evaluation from the AVAGAST randomized phase III trial. Journal of clinical oncology: official journal of the American Society of Clinical Oncology 30(17): 2119-2127, 2012.

9 Wagner AD, Schneider PM and Fleig WE: The role of chemotherapy in patients with established gastric cancer. Best Pract Res Clin Gastroenterol 20(4): 789-799, 2006.

10 Wagner AD, Grothe W, Haerting J, Kleber G, Grothey A and Fleig WE: Chemotherapy in advanced gastric cancer: a systematic review and meta-analysis based on aggregate data. J Clin Oncol 24(18): 2903-2909, 2006.

11 Cunningham D, Starling N, Rao S, Iveson T, Nicolson M, Coxon F, Middleton G, Daniel F, Oates J, Norman AR and Upper Gastrointestinal Clinical Studies Group of the National Cancer Research Institute of the United Kingdom: Capecitabine and oxaliplatin for advanced esophagogastric cancer. N Engl J Med 358(1): 36-46, 2008.

12 Al-Batran SE, Hartmann JT, Probst S, Schmalenberg H, Hollerbach S, Hofheinz R, Rethwisch V, Seipelt G, Homann N, Wilhelm G, Schuch G, Stoehlmacher J, Derigs HG, HegewischBecker S, Grossmann J, Pauligk C, Atmaca A, Bokemeyer C, Knuth A, Jäger $\mathrm{E}$ and Arbeitsgemeinschaft Internistische Onkologie: Phase III trial in metastatic gastroesophageal adenocarcinoma with fluorouracil, leucovorin plus either oxaliplatin or cisplatin: a study of the Arbeitsgemeinschaft Internistische Onkologie. J Clin Oncol 26(9): 1435-1442, 2008. 
13 Wilke H, Muro K, Van Cutsem E, Oh SC, Bodoky G, Shimada Y, Sugimoto N, Lipatov O, Kim TY, Cunningham D, Rougier P, Komatsu Y, Ajani J, Emig M, Carlesi R, Ferry D, Chandrawansa K, Schwartz JD, Ohtsu A and RAINBOW Study Group: Ramucirumab plus paclitaxel versus placebo plus paclitaxel in patients with previously treated advanced gastric or gastrooesophageal junction adenocarcinoma (RAINBOW): a doubleblind, randomised phase 3 trial. Lancet Oncol 15(11): 12241235, 2014.

14 Fuchs CS, Tomasek J, Yong CJ, Dumitru F, Passalacqua R, Goswami C, Safran H, Dos Santos LV, Aprile G, Ferry DR, Melichar B, Tehfe M, Topuzov E, Zalcberg JR, Chau I, Campbell W, Sivanandan C, Pikiel J, Koshiji M, Hsu Y, Liepa AM, Gao L, Schwartz JD,Tabernero J and REGARD Trial Investigators: Ramucirumab monotherapy for previously treated advanced gastric or gastro-oesophageal junction adenocarcinoma (REGARD): an international, randomised, multicentre, placebocontrolled, phase 3 trial. Lancet 383(9911): 31-39, 2014.
15 Gampenrieder SP, Romeder F, Muss C, Pircher M, Ressler S, Rinnerthaler G, Bartsch R, Sattlberger C, Mlineritsch B and Greil R: Hypertension as a predictive marker for bevacizumab in metastatic breast cancer: results from a retrospective matchedpair analysis. Anticancer Res 34(1): 227-233, 2014.

16 Dionisio de Sousa IJ, Ferreira J, Rodrigues J, Bonito N, Jacinto $\mathrm{P}$, Marques M, Ribeiro J, Pais A and Gervásio H: Association between bevacizumab-related hypertension and response to treatment in patients with metastatic colorectal cancer. ESMO open 1(3): e000045, 2016.

17 Cancer Genome Atlas Research Network: Comprehensive molecular characterization of gastric adenocarcinoma. Nature 513(7517): 202-209, 2014.

Received August 28, 2017

Revised September 20, 2017

Accepted September 21, 2017 